J. Clin. Chem. Clin. Biochem.

Vol. 17, 1979, pp. 457-463

\title{
Künstliches endokrines Pankreas
}

\author{
Von J. D. Kruse-Jarres
}

Klinisch-Chemische und Experimentelle Laboratorien, Chirurgische Universitätsklinik Freiburg i.Br.

(Eingegangen am 5. Dezember 1978/9. Februar 1979)

\begin{abstract}
Zusammenfassung: 16 Jahre sind vergangen, seit Kadish ((1963) Trans. Amer. Soc. Artif. Org. 9, 363-367) die ersten Versuche mit der extrakorporalen Blutglucoseregulierung publizierte und damit erste Voraussetzungen schaffte, die wesentliche Teilfunktion des endokrinen Pankreas, die Regulierung der Glucosehomöostase im Blut, durch eine künstliche Maschine zu ersetzen. Fortschritte sind inzwischen vor allem auf dem Gebiet der analytischen Technologie, der elektronischen Datenverarbeitung und des Berechnungsmodus (Algorithmen) gemacht worden. Eine zufriedenstellende Insulintherapie bei problematischen Diabetikern ist zeitlich begrenzt möglich und vorteilhaft. Doch wenn auch in den letzten Jahren erstaunliche Miniaturisierungserfolge stattfanden, so erscheint weiterhin die dauerhafte oder zumindest langzeitige Implantation eines künstlichen Pankreas noch nicht realisierbar.
\end{abstract}

\section{Artificial endocrine pancreas}

Summary: 16 years ago Kadish ((1963) Trans. Amer. Soc. Artif. Org. 9, 363-367) published his first pioneering experiments with an extracorporal blood glucose regulating machine. These experiments were fundamental to the development of an artificial endocrine pancreas that replaces the natural regulator of the blood glucose. Enormous improvements have been done as far as analytical and computer technology and algorithms are concerned. Satisfactory but time-limited insulin therapy of problematic diabetics is feasable and benefical. However, in spite of a very great miniaturization of the analytical device, of the computer system, or of the insulin delivery pump, we are still far away from a durable, or at least long-term implantation of an artificial endocrine pancreas.

\section{Einleitung}

Die Individualität eines jeden Diabetikers und die schlecht zu prognostizierende Reaktion eines stoffwechselgestörten Organismus auf endogene und exogene Wirkfaktoren machen es unmöglich, ein weitreichendes und allgemeingültiges Therapiekonzept für den Diabetiker zu finden. Die ärztliche Entscheidung für den optimalen Weg der Behandlung von insulinpflichtigen Diabetikern hängt stets von der aktuellen Stoffwechselsituation ab.

Doch bewegt sich der Diabetiker zwischen 2 Fronten: Einerseits muß er mit seiner Erkrankung ein Leben lang auskommen und muß sich dem alltäglichen Leben in seinem Rhythmus anpassen; andererseits muß er sich stets einer gewissenhaften und gelegentlich sogar sehr engmaschigen Kontrolle unterziehen, um Stoffwechselentgleisungen und somit die Unterbrechung seines therapeutisch wichtigen "normalen" Alltags zu verhindern. Es ergibt sich aus dieser Situation für ihn selbst und auch für seinen Arzt der Wunsch, das defekte Organ, das
Pankreas, mit den Mitteln der modernen Technologie zu ersetzen oder aber seine Funktion von außen her zu übernehmen und zu automatisieren.

\section{Blutglucose-Monitoring}

Primäre Voraussetzung für den Ersatz eines Organs ist zunächst einmal die Erkennung der Kenngröße(n), an der oder an denen die Funktionstüchtigkeit bewertet wird. $\mathrm{Je}$ instabiler die Werte solcher Kenngrößen sind, desto notwendiger ist es, die Gesetzmäßigkeit von Konzentrationsveränderungen in einem zeitlich engmaschigen Bestimmungsverfahren zu erfassen.

Da die Blutglucose bereits unter physiologischen Bedingungen erheblichen reaktiven Schwankungen ausgesetzt ist, führt der Wunsch nach optimaler Information weg von der Stichprobenuntersuchung über eine hochfrequente Abnahmetechnik bis hin zur kontinuierlichen Analyse. 
Die Anfänge des kontinuierlichen Monitorings gehen auf das Jahr 1956 zurück, als mit dem Technicon AutoAnalyzer der erste vollmechanisiert arbeitende, mikrochemische Analysenapparat vorgestellt wurde. Kurz danach erkannten Ferrari et al. (1), daß diese Art der Analytik auch für kontinuierliche Bestimmungen geeignet war. Sie bestimmten erstmals kontinuierlich die Blutglucose am heparinisierten Hund. Nach Einführung einer geeigneten Abnahmenadel durch Ferrari (1) und später Spathis (2), deren Idee der extrakorporalen Heparinisierung noch heute Grundlage von Abnahmekathetern für die kontinuierliche Analy tik (Abb. 1) ist, lagen auch sehr bald von anderen Arbeitsgruppen erste brauchbare Ergebnisse kontinuierlichen Blutglucosemonitorings vor (3-8).

Diese Ergebnisse ermutigten erstmals 1963 Kadish (9), eine neue Methode der Úberwachung von Blutglucosewerten durch Feedback-Kontrolle mit Hilfe eines kleinen Rechners einzusetzen. Dies waren die ersten Versuche einer extrakorporalen Blutglucoseregulierung.

Eine erhebliche Verbesserung setzte 1966 nach Einführung der wesentlich spezifischeren, enzymatischen Glucose-Bestimmung mit der Glucoseoxidase/PeroxidaseMethode durch Kawerau $(10,11)$ ein. Von nun an gelang es, wesentlich differenzierteren Einblick in das Verhalten und die Feinregulierung der Blutglucose zu er- halten. Daraus resultieren beispielsweise exakte Assimilationsberechnungen für die Glucose (12) oder die diagnostische Wertung eines initialen Glucosepeaks nach plötzlicher Hyperglykämie (13). Glucoseoszillationen (14) und -rhythmen (15) ließen sich einwandfreier erfassen und konnten so eine zuverlässige Grundlage für Regulationsmuster abgeben.

Die naß-chemische kontinuierliche Blutglucosemessung hat inżwischen viele Modifikationen erfahren. Sie wurde mit hochspezifischen Verfahren soweit optimiert, daß die Reaktionszeiten vom Beginn der Blutentnahme bis hin zur Konzentrationsangabe durch Drucker oder Schreiber auf $90 \mathrm{~s}$ gesenkt werden konnten. Bei äußerst stabilen Reagenzien wurde eine akzeptabel niedrige Störanfälligkeit des genannten Analysensystems erreicht (Abb. 2).

Ununterbrochene Messungen über mehrere Tage stellen schon lange keine Seltenheit mehr dar. Diese Grundbedingungen sind unabdingbare Voraussetzung für ein künstliches Pankreas. Es kann jedoch nicht als optimal angesehen werden, wenn dieser Art von Analytik Grenżen gesetzt werden dadurch, daß das abgenommene Blut $(2,5 \mathrm{ml} / \mathrm{h})$ verbraucht wird. Es steht dem Organismus nickt mehr zur Verfügung, was auf die Dauer eine Belastung darstellt.

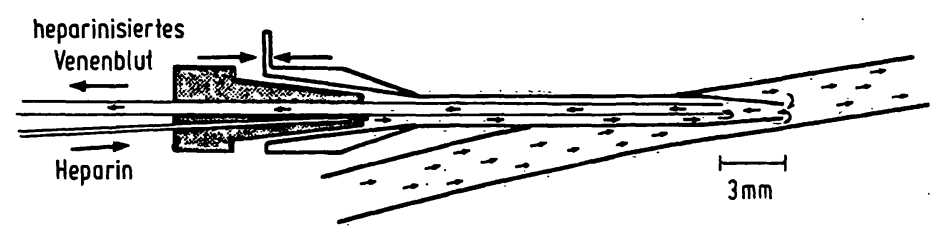

Abb. 1. Kanüle für kontinuierlichen Blutentnahme. Heparin wird zwischen dem Kanülenmantel vorgetrieben und mit dem angesaugten Venenblut an der Katheterspitze in konstantem Verhältnis gemischt.

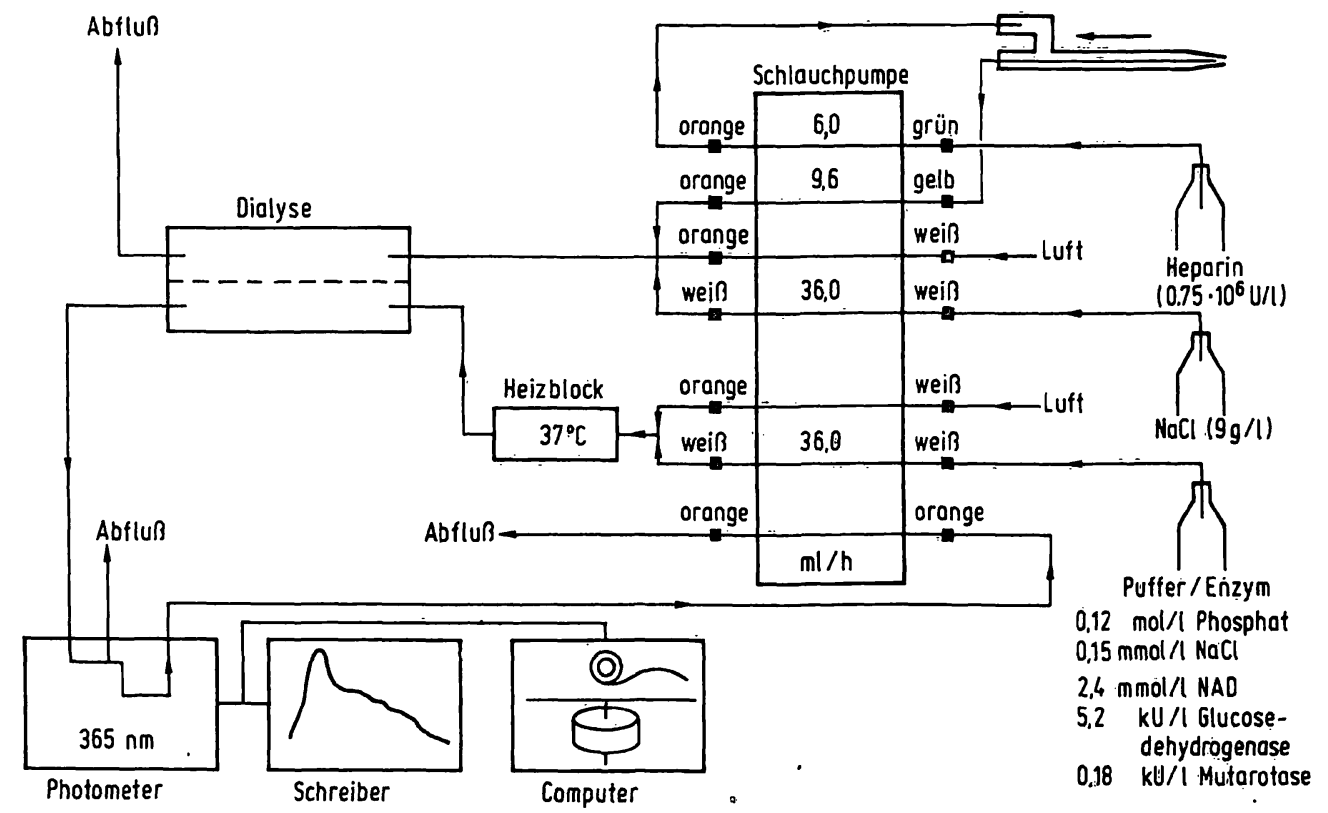

Abb. 2. Flußdiagramm für die naß-chemische, kontinuierliche Bestimmung der Blutglucose in einem geschlossenen Schlauchsystem. Die Reaktionszeit von der Abnahme des venösen Blutes bis zum Zeigerausschlag auf dem Schreiber beträgt bei. Verwendung der Glucosedehydrogenase-Methode und spezieller Schlauchkombinationen in der Pumpe $90 \mathrm{~s}$. 
Clark \& Lyons (16) entwickelten 1962 eine $\mathrm{O}_{2}$-Elektrode, bestehend aus zwei Glucoseoxidase-beschichteten Platinkathoden und einer gemeinsamen $\mathrm{Ag} / \mathrm{AgCl}$-Anode in einer Elektrolytlösung. Diese Elektrode ist Grundlage für eine Anzahl von elektrochemischen Glucosesensoren, die auch für die kontinuierliche Blutglucose-Registrierung im Blut Verwendung finden (Abb. 3). Primär für den Gebrauch der Glucosemessung im Gewebe, jedoch ebenfalls mit der Zielsetzung der Blutglucosebestimmung entwickelten Bessman \& Schultz (17) einen Glucosesensor, der ohne enzymatische Katalyse arbeitet. Die Oxidation läuft direkt an einem Katalysator aus einer Edelmetall-Legierung ( 0,85 Gold, 0,15 Platin) ab, welche der Membran einer gewöhnlichen $\mathrm{Pt} / \mathrm{KCl} / \mathrm{AgCl}-\mathrm{O}_{2}$-Elektrode aufsitzt. Der eigentliche Sensor besteht aus einer gewöhnlichen $\mathrm{O}_{2}$-Elektrode.

Ebenfalls eine katalytische Metallelektrode wurde in der Arbeitsgruppe um Soeldner (18) entwickelt. Es handelt sich um ein Meßsystem mit einer semipermeablen Membran-überzogenen Brennstoffanode für die Glucoseoxidation, einer Gegenelektrode, die von der Anode durch eine Ionenaustauschermembran getrennt ist, und einer Referenzelektrode. Dieser knopfgroße Sensor ist äußerst selektiv und kann leicht und schnell regeneriert werden.

Alle diese Sensormethoden haben eine zunehmende Bedeutung im Hinblick auf eine schnelle und präzise Meßwertdarstellung auf dem Gebiet der kontinuierlichen Blutglucoseanalytik. Sie erfüllen eine wesentliche Voraussetzung für eine computergesteuerte Blutglucose-

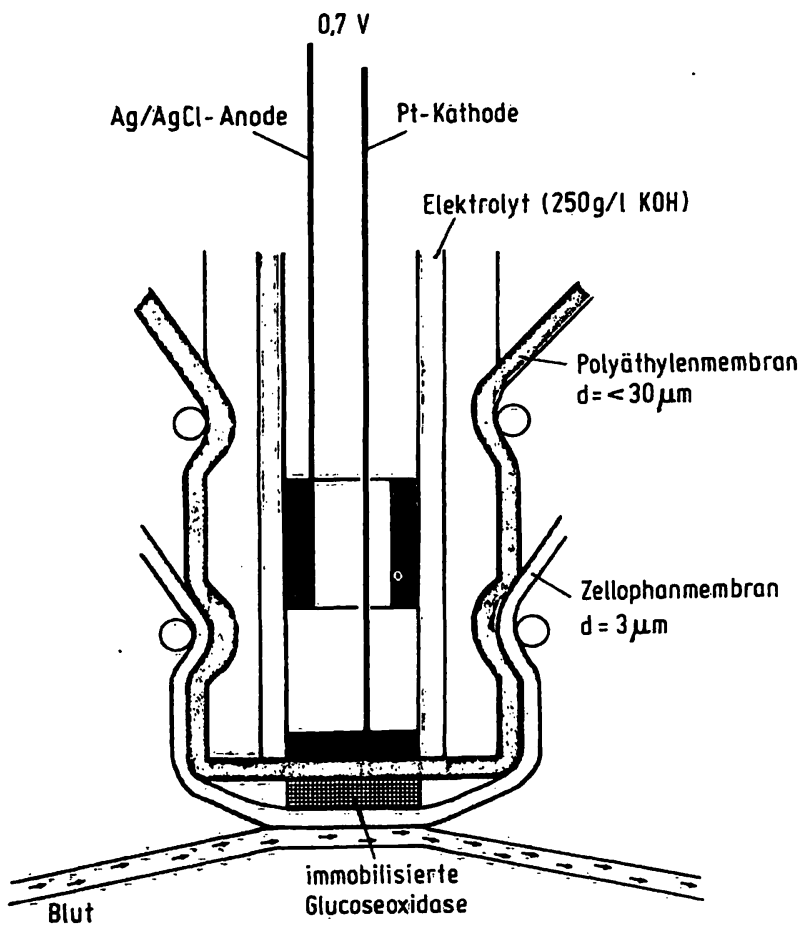

Abb. 3. Schematische Darstellung eines Glucose-spezifischen Sensors. regulierung. Sie sind zumindest in vitro durch eine hervorragende Meßgenauigkeit und eine vorteilhaft schnelle Ansprechgeschwindigkeit, sowie eine gute Meßwertkonstanz bei weitreichender Linearität gekennzeichnet.

Es bestehen allerdings noch erhebliche Schwierigkeiten bei der Daueranwendung solcher kontinuierlich messender Sauerstoffelektroden. Schranken sind darüber hinaus noch gesetzt durch die begrenzte Anwendbarkeit im Vollblut, da das Auftreten einer Drift oder einer Nicht-Linearität durch Proteinfilmbildung oder Fibrin- und Thrombocytenanlagerungen und die damit verbundenen $\mathrm{Em}$ pfindlichkeitseinschränkungen bisher nicht ausreichend beseitigt werden konnte. Daraus resultiert eine vor allem zeitlich begrenzte Verwendbarkeit. Dennoch: Das Vertrauen in die Uberwincung solcher technischer Probleme rechtfertigt die Uberzeugung, daß derartige Blutglucosesensoren die Mittel der Wahl für die Meßwerterfassung eines künstlichen endokrinen Pankreas sind.

\section{Extrakorporale Blutglucoseregulierung}

Die zunehmende Erfahrung durch kontinuierliche Glucosemessungen unterstrich immer mehr die Ansicht, daß die übliche Insulintherapie mit wenigen intermittierenden Insulininjektionen keine optimale Substitution sein kann, sondern nur eine „Prothesentherapie“ ist (Abb. 4). Unvorhergesehen gemessene und von Patienten kaum bemerkte latent hypoglykämische Stoffwechselsituationen ebenso wie starke Schwankungen der Blutglucosekonzentration zeigten an, daß Glucose- und Insulinkonzentrationen keineswegs immer sinnbildlich aufeinander abgestimmt sind.

Dennoch erwies sich eine Glucoseimbalance stets als eine Funktion von Amplitude plus Frequenz der Insulinsekretion bzw. -substitution. Diese mathematische Beziehung bedurfte exakter Formulierungen, die später die Grundlage verschiedener Algorithmen für eine zufriedenstellende Blutglucoseregulierung werden sollten. Dabei kristallisierten sich im wesentlichen 2 Prinzipien heraus: Das "closed-loop system", in welchem sich die zu substituierende Insulinmenge an der aktuellen Blutglucose orientiert und die Funktion eines geschlossenen Regelkreises erfüllt wird (Abb. 5); und das "open-loop system", in welchem nach vorheriger Erfassung eines Blutglucoseprofils eine prä-programmierte Insulininfusion unabhängig von der aktuellen Blutglucose durchgeführt wird.

Die ersten Ansätze einer Entwicklung in Richtung „Künstliches Pankreas“ sind 1963 bei Kadish (9) zu finden, der mittels eines einfachen, automatischen Feedback-Kontrollsystems versuchte, die Glucosekonzentration im Blut zwischen 3 und $8 \mathrm{mmol} / \mathrm{l}$ zu halten. Es konnte ihm auf diese Weise jedoch nicht gelingen, eine zufriedenstellende Glucosehomöostase zu erreichen. Eine Einstellung der Blutglucose mit der ausschließlichen Orientierungshilfe oberer und unterer Schwellenwerte mußte sich spätestens nach Uberwachung der 


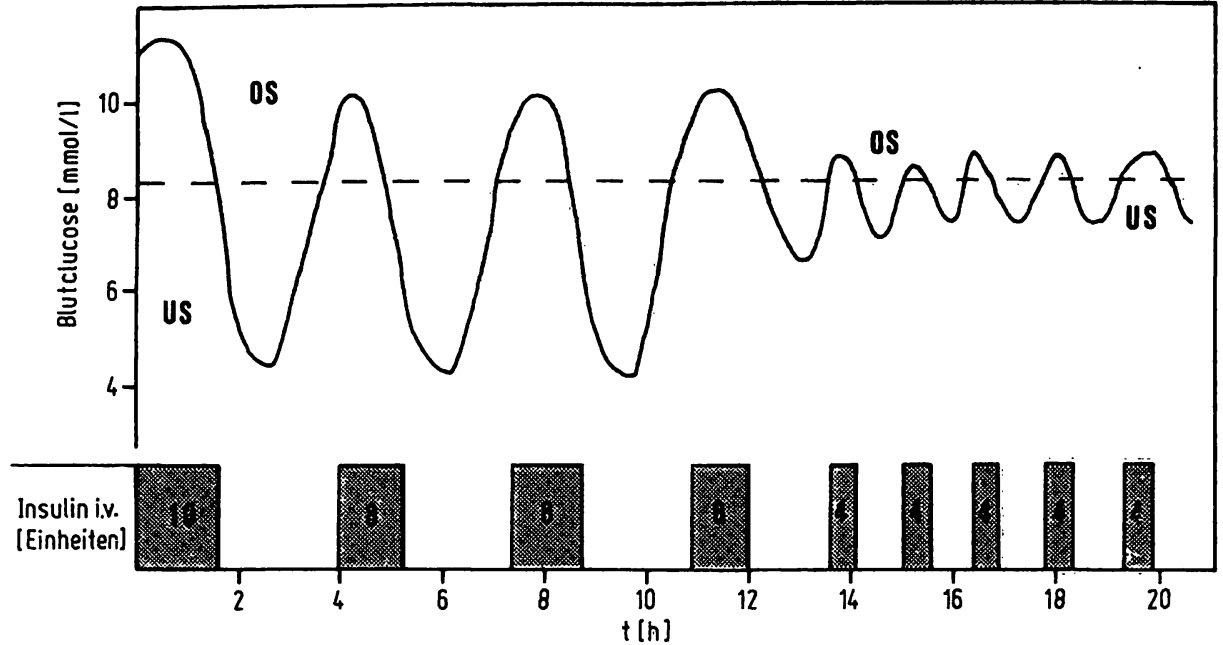

Abb. 4. Blutglucoseoszillationen eines pankreatektomierten Hundes infolge starrer und durch obere (OS) und untere (US) Schwellenwerte ausgelöster bzw. beendeter Insulininfusionen. .

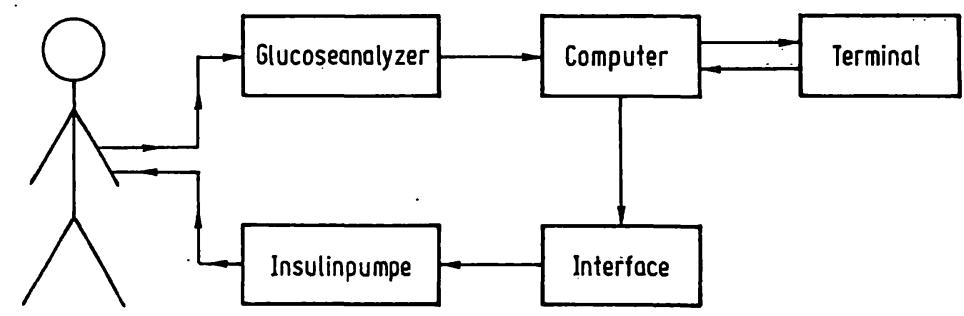

Abb. 5. Blockschaltbild einer extrakorporalen Blutglucoseregulierung.

Blutglucose mit kontinuierlichẹn Methoden über längere Zeiträume (Glucose-Langzeitprofile (19)) als unbrauchbar erweisen.

1973 haben erstmals Albisser et al. (20) eine Versuchsanordnung einer extrakorporalen Blutglucoseregulierung mit brauchbaren mathematischen Algorithmen für die Ansteuerung von Glucose- und Insulinpumpen vorgestellt. Die reaktive Insulingabe stellte dabei eine hyperbolische Funktion der Richtung der Blutglucosekonzentrationsveränderung dar (Abb. 6 links oben). So
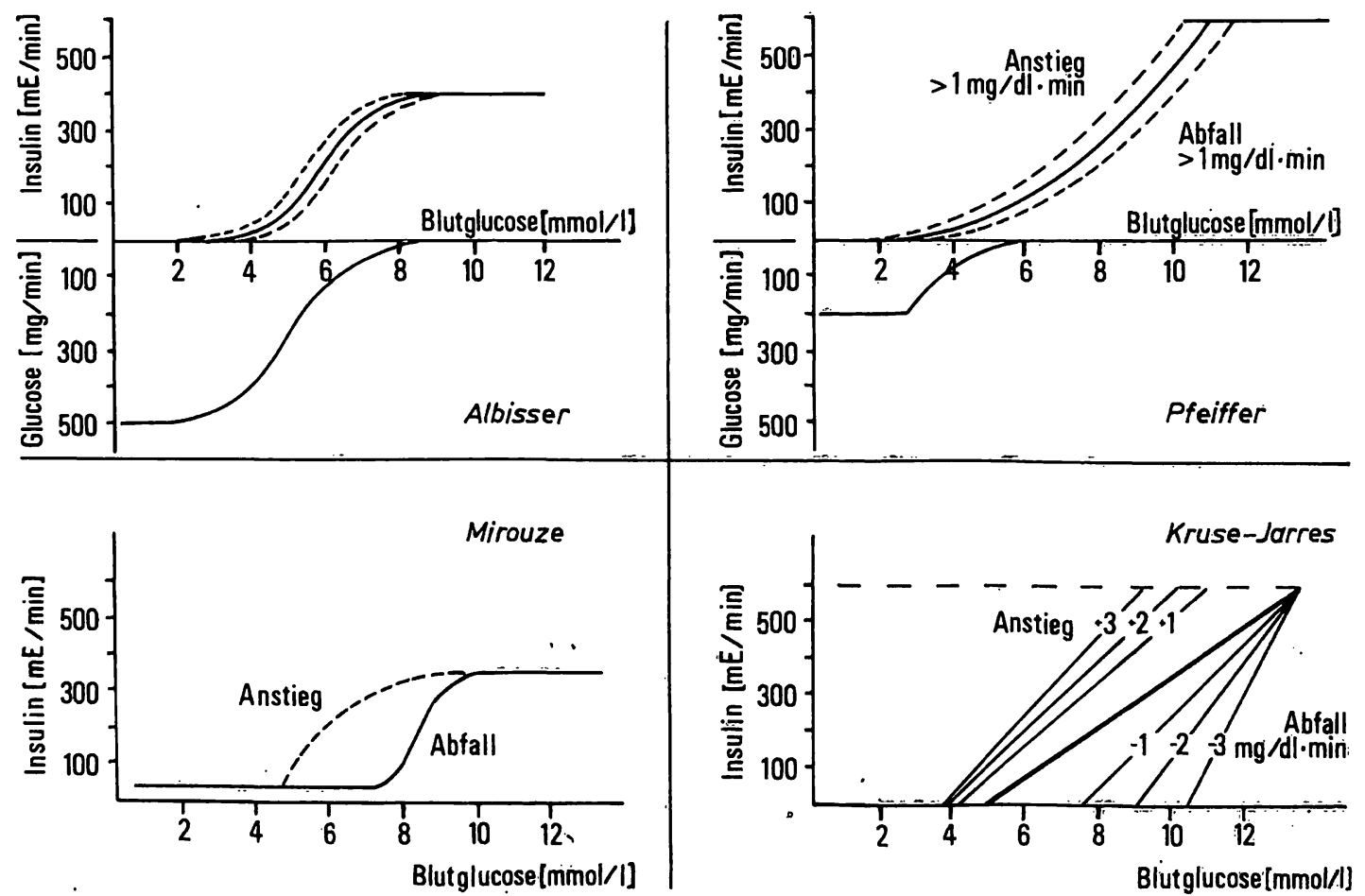

Abb. 6. Kennlinien der Steueralgorithmen zur extrakorporalen Blutglucoseregulierung. Siehe Text. 
konnte eine normoglykämische Stoffwechsellage am pankreatektomierten Hund erreicht werden. Zudem gestattete die Programmierung nicht nur eine monophasische Insulinfreisetzung in proportionaler Abhängigkeit von der verzögert gemessenen Blutglucosekonzentration, sondern auch eine den physiologischen Gegebenheiten angepaßte biphasische Steuerung (21). Dadurch wurden reak tive Hypoglykämien und starke Blutglucoseschwankungen verhindert. Der Insulinverbrauch sank um bis zu $90 \%$. Durch diesen verbesserten Kontrollalgorithmus konnte fortan auf die Gegensteuerung mit Glucose oder Glucagon verzichtet werden.

Die Arbeitsgruppe um Pfeiffer (22) untersuchte ebenfalls seit 1973 zunächst naß-chemisch, später mit der Sensortechnik (Biostator ${ }^{\circledR}$ ) die proportionale Abhängigkeit der Insulininfusionsrate von der Blutglucosekonzentration und kombinierte sie mit der Derivativkontrolle (23) am Menschen. Der Computer ist dabei so programmiert, daß er den Trend der Blutglucose berücksichtigt, d.h. er richtet sich nach einer extrapolierten, vorausberechneten Glucosekonzentration auf der Basis von Gleichungen 4. Ordnung (Abb. 6 rechts oben).

Im Gegensatz zu den beiden beschriebenen System arbeitet das ,aep“ (artificial endocrine pancreas) aus der Arbeitsgruppe von Kruse-Jarres (24) zwar auch nach dem Prinzip des closed-loop system, jedoch mit Algorithmen, die der Regeltechnik entlehnt sind und auf einer P/DRegelung (Proportional/Differential) beruhen (Abb. 6 rechts unten). Im Minutentakt wird unter Berücksichtigung von Glucoseabbau, Insulinhalbwertszeit und Zeitveržug zwischen Blutabnahme und Insulinwirkung ein Berechnungszyklus durchgeführt, der sich in einem Kurzzeit- oder Langzeitsystem entsprechend der Geschwindigkeit der Glucoseverändenung laufend wiederholt. Die Entscheidungen von seiten des Rechners fallen aufgrund von digital umgesetzten Tabellen analog zu den Kennlinien der Proportionalsteuerung und aufgrund von Glucoseäquivalenztabellen, die die Insulinwirkung auf die Glucose in Abhängigkeit von der Zeit wiedergeben.
Im Hinblick auf die forcierten Bemühungen um methodisch-optimale Lösungen für eine extrakorporale Blutglucoseregulierung seien hier vor allem auch die Arbeitsgruppen um Berg \& Sailer (25), Deckert (26), Genuth (27), Hepp (28), Irsigler (29), Kohlendorf (30), Mirouze (Abb. 6 links unten) (31) und Slama (32) genannt. Alle arbeiten zum Teil mit eigenen Algorithmen entweder nach dem Prinzip des geschlossenen Systems mit Feedback-Mechanismen (closed-loop system) oder mit präprogrammierten Insulininfusionssystemen (open-loop system).

Die extrakorporale Blutglucoseregulierung findet neben der klinisch-experimentellen Forschung ihre klinischen Anwendungsgebiete vornehmlich bei den Stabilisierungsversuchen labiler Diabetiker, bei der Behandlung des Coma diabeticum und bei der Úberwachung diabetischer Patienten während chirurgischer und geburtshilflicher Eingriffe. Vor allem die klinischen Einsätze bei der Therapie labiler diabetischer Stoffwechselsituationen unter und nach operativen Eingriffen (Abb. 7) und nach schweren Traumata (Abb. 8) haben deutlich gezeigt, daß sich die Blutglucose über ein artifizielles Pankreas extrakorporal sowohl aus dem pathologischen in einen physiologischen Bereich, als auch innerhalb des physiologischen Bereich zufriedenstellend steuern läßt; und dies im allgemeinen ohne Gegenregulation mit Glucose, d.h. ohne Erzeugung hypoglykämischer Situationen. Es erscheint so gerechtfertigt, bedingt von einer ,künstlichen $\beta$-Zelle " $z u$ sprechen.

Im Gegensatz zu zweifelsohne vorteilhaften, jedoch fest verdrahteten Mini- und sogar Mikroprozessoren stehen bei anderen Systemen voll programmierbare Dialogrechner zur Verfügung, die zusätzlich alle Möglichkeiten der Weiterentwicklung und Modifikation bieten. Der relativ umfangreiche EDV-Aufwand erweitert das Einsatzgebiet und erleichtert die Anpassung an jeden diabetischen Problempatienten, stellt jedoch an den Operator weniger in der Handhabung als vielmehr in der Vielfalt der Entscheidungsmöglichkeiten größere Anforderungen. Insofern gilt es bei der weiteren Entwicklung des Pro-

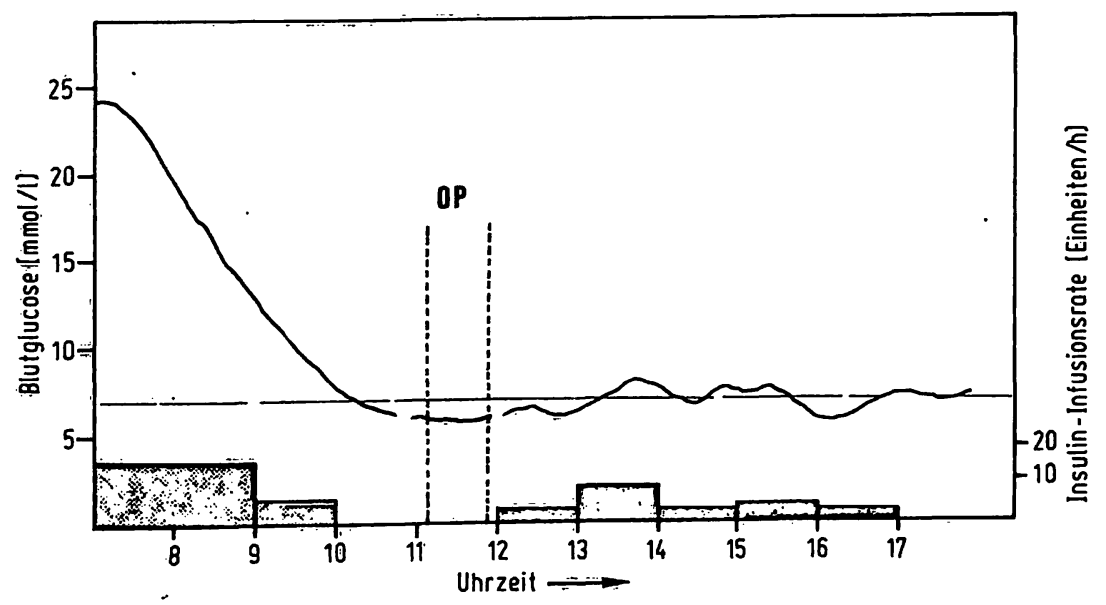

Abb. 7. Glucogramm und Insulin-Infusionsrate vor, während und nach Dünndarmresektion bei bestehendem manifesten, Insulinbedürftigen Diabetes. 


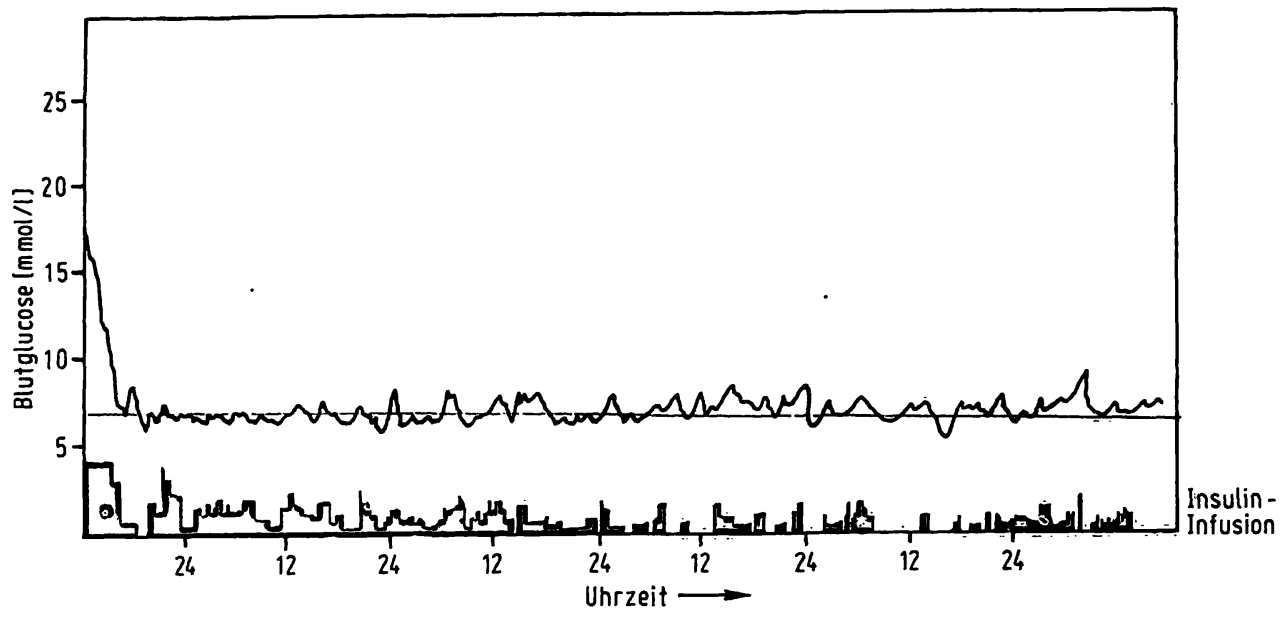

Abb. 8. 52 Jahre alter, bewußtloser Patient (W.M.) nach schwerem Schädel-Hirn-Trauma unter konstanter intravenöser Glucosezufuhr von $200 \mathrm{~g} / \mathrm{d}$ und automatischer extrakorporaler Blutglucoseregulierung mit dem ,aep“. (Schraffierte Bereiche: Nächte).

blems zu lösen, dem Platzmangel und der Hektik z.B. von Intensivstation und Operationssaal durch flexible Komprimierung von soft- und hardware zu begegnen.

\section{Implantierbare künstliche $\beta$-Zelle}

Eine weitere Miniaturisierung führt in die Richtung des ursprünglichen Wunsches, über ein transportables „Rucksackgerät“" oder das ,,Steuerungsgerät in der Jackentasche" zu einem implantierbaren Regler zu kommen. Die Schwierigkeiten bei der Konstruktion eines geeigneten Fühlers für die Blut- oder Gewebsglucose wurden bereits erwähnt. Hier steht momentan weniger die Schwierigkeit bei der Miniaturisierung eines geeigneten Sensors (33) im Vordergrund der Probleme zur Implantation, als vielmehr die Unverträglichkeit und die begrenzte Funktionstüchtigkeit (34). Methoden der Laser-Absorptionsspektroskopie (35) sind leider zur Zeit noch für Implantationszwecke utopisch.

Erfolgreicher war bisher die Arbeit an geeigneten, miniaturisierten und gut steuerbaren Insulininfusionspumpen. Sowohl aus den Arbeitsgruppen von Bessman (36) und von Shumakov (37) als auch von Franetzki (38) und von Irsigler (39) wurden bereits Modelle miniaturisierter Pumpeneinheiten vorgestellt, die im Rahmen des prä-programmierten open-loop system bereits klinisch Anwendung finden. So ist es mit einer solchen miniaturisierten Pumpe und einem elektronischen Versorgungsgerät bereits zuverlässig möglich, den Diabetiker entšprechend seinem wechselnden Bedarf Insulin in winzigen Raten (Größenordnung von $\mu \mathrm{l} / \mathrm{h}$ ) zuzuführen. Die Dosiereinrichtung enthält neben der Pumpe den Insulinspeicher, die Energiequelle für mindestens 2 Jahre und einige elektronische Kreise. Ein weiteres Gerät liefert die Signale für die Pumpe, zeigt die abgegebene Insulinmenge an und überwacht deren Funktion.

Sicherlich ist der Weg noch weit bis zur Realisierung des Wunschtraums von einem transplantierbaren künstlichen Pankreas (Abb. 9), wie es von vielen angestrebt wird (40).
Mit den bisherigen Lösungen einer extrakorporalen Blutglucoseregulierung werden jedoch bereits hoffnungsvolle erste Schritte für eine zufriedenstellende Therapie problematischer Glucoseentgleisungen getan. Deutliche Insulinreduktionen bei der Subștitutionstherapie sind die Folge einer künstlichen Sekretion, die die physiologischen Verhältnisse entsprechend der aktuellen Anforderung nachahmt.

Wie immer auch die ,künstlichen Pankreata“ der verschiedenen Arbeitsgruppen aussehen: Das Problem der Zukunft liegt nicht mehr so sehr in dem know how der Aufrechterhaltung bzw. Erzielung der Blutglucosebalance, und auch nicht so sehr in der Miniturisierung der gesamten Apparatur; im Vordergrund muß vielmehr bald eine Antwort auf die Frage stehen, welches Kompartiment fựr die Messung der Glucose und die Zufuhr von substituiertem Insulin sowohl aus physiclogischem als auch aus technischem Blickwinkel des geeignetste ist, und ob nicht der Insulinkinetik im Blut bei der Blutglucoseregulierung eine größere Bedeutung zugeschrieben werden muß. In der Beurteilung verschiedener Gewebe oder des Blutes als dem repräsentativen Organ für die Implantation bzw. die Applikation eines künstlichen endokrinen Pankreas scheiden sich vorerst noch die Geister. Ein Insulinmonitoring (41) scheitert für die Zwecke

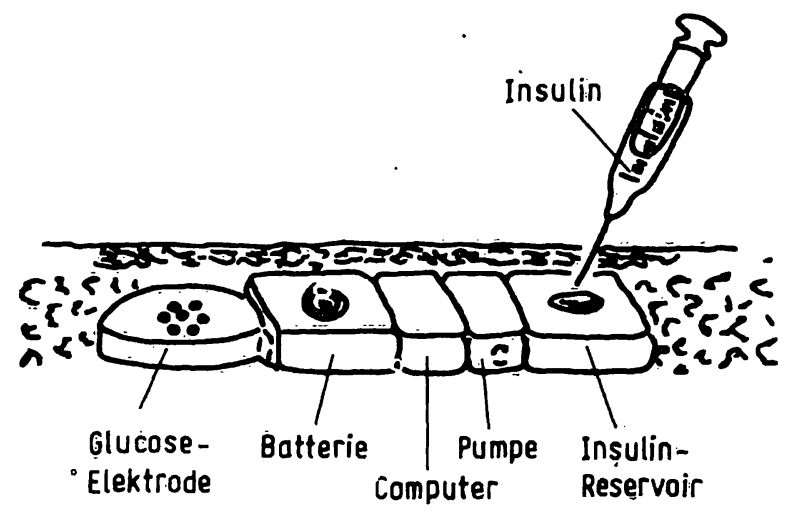

Abb. 9. Bestand teile einer total implantierten künstlichen $\beta$ Żelle (nach 1.c. (40)). 
eines closed-loop system zunächst noch an den technischen Voraussetzungen. Mit großer Sicherheit bringt die Technik des kontinuierlich aufgezeichneten Insulinprofils für ein künstliches endokrines Pankreas wichtige neue Informationen und vielleicht Modifikationen der Steueralgorithmen.

\section{Literatur}

1. Ferrari, A., Kessler, G., Russo-Alesi, F. M. \& Kelly, J. M. (1960), Ann. N.Y. Acad. Sci. 87, 729-744

2. Spathis, G. S., Hurst, R. J. \& Pollard, A. (1966), Lancet $I J$, 266-267

3. Burns, T. W., Bregant, R. \& van Peenan, H. J. (1963), J. Lab. Clin. Med. 62, 863

4. Castaigne, P.. Cambier, J. \& Schuller, E. (1963), Presse Méd. $71,2555-2557$

5. Galli, A., Jeanmaire, J., Choisy, H. \& Schuller, E. (1961), Ann. Biol. Clin. 19, 559-577

6. Mirouze, J., Monnier, C., Sany, C., Jaffiol, C. \& ArnavielhieBony, M. (1962), Ann. Biol. Clin. 20, 1019-1029

7. Verdeaux, G., Schuller, E. \& Bonnafé, M. (1959), Rev. Neurol. 101, 276-278

8. Weller, C., Linder, M., Macauley, A., Ferrari, A. \& Kessler, G. (1960), Ann. N.Y. Acad. Sci. 87, 658-668

9. Kadish, A. H. (1963), Trans. Amer. Soc. Artif. Int. Org. 9, 363-367

10. Kawerau, E. (1966), Z. Klin. Chem. Klin. Biochem. 4, 224232

11. Kawerau, E. \& Surtecs, S. J. (1966), Z. Klin. Chem. Klin. Biochem. 4, 237-247

12. Kruse-J arres, J. D. (1972), Med. Welt. 23, 50-54

13. Kruse-Jarres, J. D., Reiter, J. \& Klingmüller, V. (1969), Klin. Wochenschr. 47, 469-475

14. Kruse-Jarres, J. D. (1969), Klin. Wochenschr. 47, 10791084

15. Kruse-Jarres, J. D. (1974), Z. Klin. Chem. Klin. Biochem. 12,43

16. Clark, L. C. \& Lyons, C. (1962), Ann. N.Y. Acad. Sci. 102, $29-46$

17. Bessman, S. P. \& Schultz, R. D. (1972), Horm. Metab. Res. 4, 413-417

18. Chang, K. W., Aisenberg, S., Soeldner, J. S. \& Hiebert, J. M. (1973), Trans. Amer. Soc. Artif, Int. Org. 19, 352-360

19. Kruse-Jarres, J. D., Hilpert, C. \& Klingmüller, V. (1972), Med. Klin. 67, 226-230

20. Albisser, A. M., Leibel, B. S., Ewart, T., Davidovac, Z. \& Zingg, W. (1973), Diabetes 22, Suppl. 1, 294

21. Albisser, A. M., Leibel, B. S., Ewart, T., Davidovac, Z., Botz, C. K. \& Zingg, W. (1974), Diabetes 23, 389-396

22. Pfeiffer, E. F. \& Thum, Ch. (1974), Naturwissenschaften 61 , $455-456$
Solange solche Fragen keine endgültige Antwort erfahren, werden vielschichtige technische Details verschiedener Verfahren im Vordergrund stehen. Die alleinige Konzentration auf die Technologie wird jedoch die notwendige Klärung wesentlicher pathobiochemischer Grundsatzfragen verzögern. Sehr vieles ist noch zu tun!
23. Pfeiffer, E. F., Thum, Ch., Beischer, W. \& Clemens, A. H. (1975), Verh. Dtsch. Ges. Inn. Med. 81, 602-615

24. Kruse-Jarres, J. D., Braun, G., Bresch, M., Lehmann, U., Letulé, U. Naegele, R. \& Winkelmann, R. (1979), Med. Welt. 30, 83-88

25. Berg, G. \& Sailer, D. (1977), Horm. Metab. Res. Suppl. No. $7,41-53$

26. Deckert, T. \& L $\phi$ rup, B. (1976), Diabetologia 12, 573579

27. Genuth, S. \& Martin, P. (1977), Diabetes 26, 571-581

28. Hepp, K. D., Renner, R., von Funcke, H. J., Mehnert, H., Haerten, R. \& Kresse, H. (1977), Horm. Metab. Res. Suppl. No. 7, 72-76

29. Irsigler, K., Kritz, H., Koller, W., Kaspar, L., Mader, R. \& Franetzki, M. (1978), Med. Klin. 73, 1738-1743

30. Kolendorf, K. \& Bojsen, J. (1979), Horm. Metab. Res. (im Druck)

31. Mirouze, J., Selam, J. L., Pham, T. C. \& Cavadore, D. (1977), Diabetologia $13,273-278$

32. Slama, G., Hautecouverture, M., Assan, R. \& Tschoubroutzky, G. (1974), Diabetes 23, 732-738

33. Bessman, S. P. (1974), Metabol. Ther. 3, 4

34. Gough, D. A., A isenberg, S., Colton, C. K., Giner, J. \& Soeldner, J. S. (1977), Horm. Metab. Res. Suppl. No. 7, 10-22

35. Kaiser, N. (1979), Horm. Metab. Res. (im Druck)

36. Thomas, L. J. \& Bessman, S. P. (1975), Trans. Amer. Soc. Artif. Int. Org. 21, 516-520

37. Shumakov, V. (1975), Trans. Amer. Soc. Artif, Int. Org. 21, $521-522$

38. Franetzki, M., Prestrele, K. \& Kresse, H. (1978), Biomed. Tech. 23, 163-164

39. Kritz, H., Brändle, J., Koller, W., Franetzki, M. \& Irsigler, K. (1978), in: Diabetes-Probleme in der Schwangerschaft (Hrsg. K. Irsigler), Urban \& Schwarzenberg, München-Wien 81-89

40. Soeldner, J. S., Chang, K. W., Aisenberg, S. \& Hiebert, J. M. (1973), in: Temporal Aspects of Therapeutics (J. Urquhart \& F. E. Yates, eds.), Plenum Press, New York-London, 181207

41. Trendelenburg, Chr., Daniel, P., Wieland, F.\& Kruse-Jarres, J. D. (1977), Horm. Metab. Res. Suppl. No. 7, 34-40
Prof. Dr. J. D. Kruse-Jarres

Klin. Chem. und Experiment. Lab.

Chirurg. Univ. Klinik

Hugstetterstraße 55

D-7800 Freiburg i.Br. 


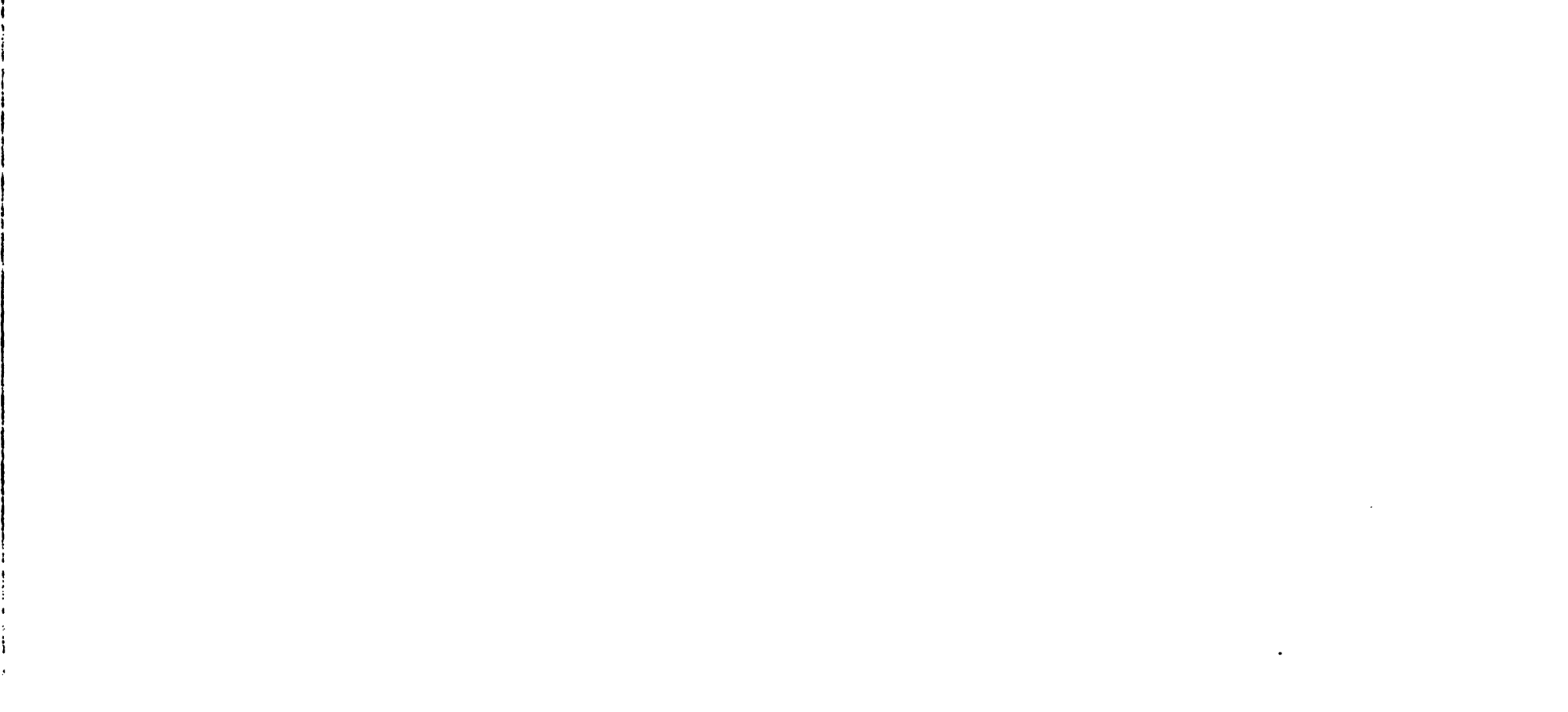

